\title{
Colocalization Analysis of PPP1 Isoforms and Two Novel Targeting Subunits in Breast Carcinoma
}

\author{
C. Sousa* ${ }^{1}$, A. P. Vintém ${ }^{1}$, M. Fardilha ${ }^{1}$, O. da Cruz e Silva ${ }^{2}$ and E. da Cruz e Silva ${ }^{1}$ \\ ${ }^{1}$ Signal Transduction Lab, CBC and Department of Biology, University of Aveiro, Campus de \\ Santiago, 3810-193 Aveiro, Portugal \\ ${ }^{2}$ Neurosciences Lab, CBC, University of Aveiro, Campus de Santiago, 3810-193 Aveiro, Portugal
}

\begin{abstract}
Protein phosphatase 1 (PPP1) is the PPP most ubiquitous and each isoform interact with regulatory subunits that may be responsible for their subcellular localization. We identified PPP1R15B, C1ORF71 as novel regulators and the aim of this study was their further characterization in carcinoma cells. We analysed localization of each regulator in MDA-MB-468 cells and we transfected with constructs that we made with each as a GFP-fusion protein. For PPP1 cellular localization we used specific antibodies for each isoform. We observed the cells under a fluorescent microscope and LSM and we quantified co-localization. We found a high overlap coefficient of both the novel proteins with PPP1alpha and PPP1gamma1. We propose a model in which PPP1 regulator interacts with one or two regulatory subunits that may be used as target for therapeutic strategies.
\end{abstract}

KEY-WORDS

Fluorescent microscopy, laser scanning microscopy, overlap coefficient, PPP1, novel regulators, breast carcinoma

\section{ACKNOWLEDGMENTS}

This work was supported by FCT: CS received a PhD fellowship, and A.P.V. and M.F. received pos-doctoral fellowships.

\section{INTRODUCTION}

Protein phosphorylation/dephosphorylation is a reversible process that enables cells to respond to several different signals in a dynamic way.

PPP1 (protein phosphatase 1) is the PPP most ubiquitous: we can find several PPP1 isoforms that are expressed by different genes, such as PPP1CA, PPP1CB, PPP1CC. This late gene is responsible for two transcripts, PPP1gamma1 and PPP1gamma2, by alternative splicing [1]. All these isoforms have different tecidular localization and are known to interact with several regulatory subunits at the subcellular level.

It is accepted that the regulatory subunits of PPP1 are responsible for the regulation of the activity of this enzyme, as well as by its particular subcellular localization.

We identified PPP1R15B, C1ORF71 as novel regulators from yeast two-hybrid screens both using cDNA from brain and testis [1] and the aim of this study was their further characterization in carcinoma cells. 


\section{MATERIALS AND METHODS}

Cloning: Standard molecular biology was done. Briefly, PPP1R15B was PCR cloned into a pEGFPN1 vector with appropriate primers with $S a c$ I and $S a l$ I restriction sites. C1ORF71 was cloned into a pEGFP-N1 vector with appropriate primers with appropriate primers with $\mathrm{XhoI}$ and $\mathrm{XmaI}$ sites.

Samples: Slides of fixed MDA-MB-468 (ATCC HTB-132) transfected cells (with either PPP1R15B-GFP or C1ORF71-GFP). Cells were grown on glass coverslips coated with polyornithine (Sigma, Portugal) and fixed in 4\% paraformaldehyde and pure methanol (Sigma, Portugal). Antibody staining was performed as previously described [2]. Briefly, slides were incubated at room temperature with primary antibodies: anti-PP1gamma antibody (Covalab, UK) at 1:100, anti-gamma2 (G502) at 1:500 and anti-PPP1R15B (Covalab, UK) and anti-C1ORF71 (Covalab, UK) both at 1:50 dilution. After washing in PBS, Texas red-conjugated goat anti-rabbit secondary antibodies (Molecular Probes, USA) were added for $2 \mathrm{~h}$ at 1:300 dilution. DNA was counterstained with DAPI in the Vectorshield mounting media (Vector, USA).

Imaging: Epifluorescent microscopy and confocal laser scanning microscopy (CLSM) were used. For each sample, in CLSM, a stack of images was acquired, each in a slightly different focal plane, and a 3D representation of the sample was obtained. The microscopes used were a Zeiss CLSM 510 and an Olympus IX-81 epifluorecent microscope available at the University of Aveiro. The images in the stacks were acquired using a $60 \times$ objective. We used specific software in order to quantify co-localization analysing several different cells and slides.

\section{RESULTS AND DISCUSSION}

We found PPP1alpha in the nucleus with a diffuse pattern; PPP1gamma1 in the nucleus, outside the nucleolus, and in the cytoplasm; and PP1gamma2 is almost completely absent from these cells, as reported by others (Figs.1 and 2). Our report is the first one, as far as we know, of the localization of these new regulators, that is PPP1R15B is distributed in both cytoplasm and nucleus (less) and C1ORF71 has a a particular "ring-like" distribution around the nucleus, what we suggested that might be ER. We colocalized these new proteins with PPP1 isoforms (Figs.1 and 2).

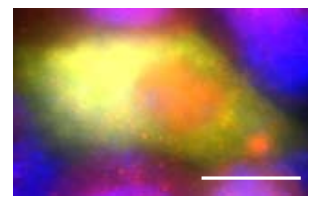

Fig.1 (a)

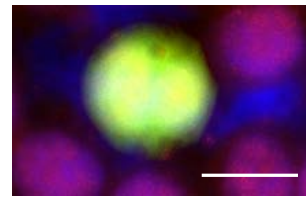

b)

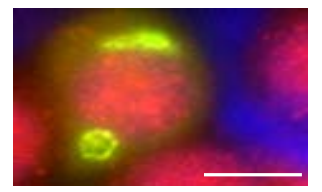

Fig.2 (a)

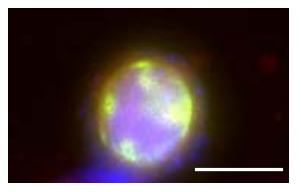

(b)

Fig. 1 - Subcellular distribution of PPP1R15B-GFP in breast carcinoma cells by epifluorescence microscope: (a) colocalization with PPP1alpha and (b) colocalization with PPP1gamma1. In green channel is PPP1R15B-GFP, in the red channel is anti-PPP1 isoform (Texas Red) and in the blue channel is DAPI nuclear staining (Scale bar $=20 \mu \mathrm{m}$ ).

Fig. 2 -. Subcellular distribution of C1ORF71-GFP in breast carcinoma cells by epifluorescence microscope: (a) colocalization with PPP1alpha and (b) colocalization with PPP1gamma1. In green channel is C1ORF71-GFP, in the red channel is anti-PPP1 isoform (Texas Red) and in the blue channel is DAPI nuclear staining (Scale bar $=20 \mu \mathrm{m})$. 


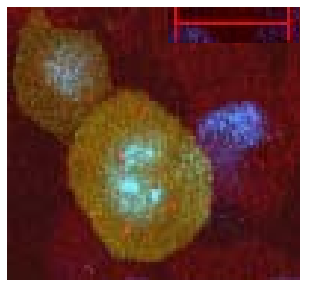

Table 1 - Overlap coefficient of PPP1R15B-GFP and C1orf71-GFP, with each PPP1 isoform in MDA-MB-468 cells (shown as percentage).

\begin{tabular}{|c|c|c|}
\hline & PPP1R15B & C1ORF71 \\
\hline PP1alpha & $40.3 \%$ & $64.7 \%$ \\
\hline PP1gamma1 & $34.5 \%$ & $62.0 \%$ \\
\hline
\end{tabular}

Fig. 3 - Subcellular distribution of PPP1R15B-GFP in carcinoma cells by CLSM: colocalization with PPP1gamma in a 3D reconstruction: in green channel is PPP1R15B-GFP, in the red channel is anti-PPP1gamma (Texas Red) and in the blue channel is DAPI nuclear staining (Scale bar $=20 \mu \mathrm{m}$ ).

We analysed the colocalization of each regulator with each PPP1 isoform (only gamma1 and alpha) and we determined the overlap coefficient of each pair (summarized in Table 1). We found good values of colocalization mainly for C1ORF71 with both isoforms and PPP1R15B mainly with PPP1alpha. This is in accordance with results of others in which they showed that in breast carcinoma PPP1alpha interacts with an important oncogene BRCA1 [3].

\section{CONCLUSIONS}

We propose a model in which PPP1 regulator interacts with one or two regulatory subunits that may be used as target for therapeutic strategies.

\section{REFERENCES}

1. Sousa, C. et al. Different subcellular distribution of novel regulators of protein phosphatase 1 Signal Transduction (submitted).

2. Faragher, A. J. and Fry, A. M., Nek2a kinase stimulates centrosome disjunction and is required for formation of bipolar mitotic spindles, Mol Biol Cell 14 (7): 2876-2889, 2003.

3. Hsu, L. C. Identification and functional characterization of a pp1-binding site in brca1, Biochem Biophys Res Commun 360 (2): 507-512, 2007. 